\title{
Technical Characteristics and Energy Performance of Double Skin Faces: A Review
}

\author{
Cui $\mathrm{Xu}^{1}$, Qiao $\mathrm{Xi}^{1}$, and Qingsong $\mathrm{Ma}^{1, *}$ \\ ${ }^{1}$ Qingdao University of Technology, College of Architecture and Urban Planning, Qingdao, Shandong, China
}

\begin{abstract}
Double Skin Facades (DSFs) have the advantages of heat preservation, heat insulation, noise reduction and energy saving. Therefore, DSFs are gradually developing into a new direction of environmental protection and energy saving of the curtain wall. This article mainly introduces the technical characteristics of DSFs from two aspects: the geometric structures of the hot aisles and the ventilation modes. And this article systematically reviews more than twenty articles which have dealt with the energy performances: thermal performances and ventilation performances. The paper finishes with some conclusions: The unique structure of DSFs makes DSFs outstanding in terms of energy saving, ventilation. However, DSFs also have some shortcomings: the initial investment and maintenance costs are relatively high, the fire protection design is relatively difficult, and the industry lacks industry standards. Therefore, it is necessary to estimate the overall energy-saving effect in advance, continue to in-depth optimization research on the engineering application value of DSFs, and ultimately bring good social and economic benefits.
\end{abstract}

\section{Introduction}

Energy crisis and environmental pollution are increasingly becoming global problems, and promoting building energy conservation and environmental protection has become a new trend in building. Compared with the traditional single-layer curtain wall, double skin facades show unique advantages in terms of thermal performance. It can not only achieve the fundamental functions as facade, such as isolating noise, keeping warm, resisting wind and rain, but also shading the sunlight, and actively using natural energy to improve energy efficiency, which represents the new development direction of curtain wall technology [1].

Double Skin Facades (DSFs), which are composed of outer facade, the intermediate cavity and inner facade. The air can be formed in order in the hot aisle (the intermediate cavity) to achieve the management of air flow. And adjustable sunshade device is arranged in the cavity. Such curtain walls are called double skin Facades This technology first appeared in developed countries such as Europe. It has developed rapidly in China in recent years and has achieved certain results. Intensifying the design of outer unit curtain walls, inner component curtain walls, thermal channels, fire protection measures, etc. can achieve good energysaving effects of double skin Facades.

\section{DSFs classified by geometric structures of hot aisle}

According to the geometric structures of hot aisles in the double skin facades, DSFs can be classified into four types: box-type, shaft-type, corridor-type, integral-type [2]. The four types given in Fig 1.
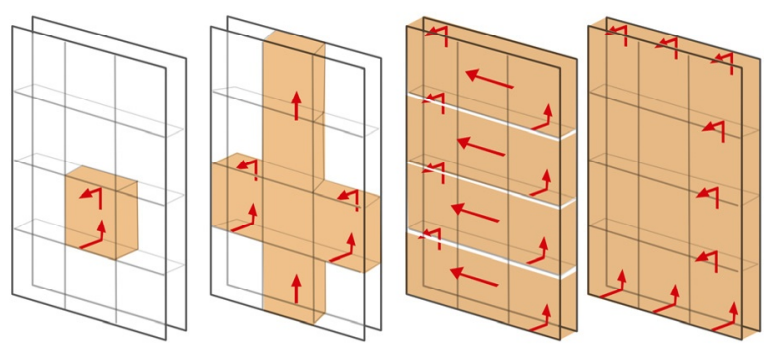

(a) Box-type

(b) Shaft-type

(c) Corridoral-type

(d) Integral-type

Fig. 1 Four types classified by geometric structures.

\subsection{Box-type}

The hot aisle of the box-type DSFs are constrained by horizontal and vertical partitions into several box-like spaces. Each window box space can span one or more floors in the longitudinal direction, and can span one or several window units or rooms in the transverse direction [2].

\footnotetext{
$\bar{*}$ Corresponding author: maqingsong@qut.edu.cn
} 
The construction of the box-type DSFs have a short construction period, does not require scaffolding, and has high fire resistance. It is especially suitable for highrise building curtain walls [3].

\subsection{Shaft-type}

The shaft-type DSFs are like a community composed of many units of box-type DSFs and ventilation shafts. Several units of box-type DSFs are attached to both sides of each ventilation shaft. In the same way, both sides of unit of box-like DSFs are respectively connected to a ventilation shaft through the upper vent on the contact surface. When the entire DSFs are doing air-organized work, each unit of box-like DSFs performs air circulation independently, and discharges air into the ventilation shaft through the upper vents on both sides of the hot aisle [2].

The shaft-type DSFs are suitable for buildings with a height of less than $20 \mathrm{~m}$. The applicability is very poor, and the application is very few [3].

\subsection{Corridor-type}

The corridor-style DSFs are divided into several long and narrow corridor-like space by several horizontal partitions. The hot aisle is constrained in the same way. Each hot aisle can span one or several floors longitudinally [2].

The corridor-type DSFs are conducive to sound insulation between floors and can prevent the spread of fire. However, it is not conducive to sound insulation and fire prevention between the same layers. Corridortype DSFs are suitable for building curtain walls with integral internal separation. The shaft-type has good vertical ventilation effect. However, the heat pressure of vertical shaft-type is too high, the temperature is too high. And the ventilation cavity is slender, which is easy to cause noise and is difficult to fire prevention [3].

\subsection{Integral-type}

The integral-type DSFs means that there is no horizontal and vertical separation, and the thermal aisle of DSFs span the entire building facade from bottom to top [2].

The integral-style DSFs are not suitable for high-rise large buildings, and the height should be less than $20 \mathrm{~m}$ [3].

Relevant data show that the largest proportion of corridor-type DSFs is $59 \%$, followed by box-type DSFs $23 \%$, corridor-type DSFs $17 \%$, and shaft-type DSFs the least, only $1 \%$ [4].

\section{Working Mechanisms of Different ventilation}

The working mechanisms of double skin facades can be classified into three types according to different ventilation methods: buffer-cavity ventilation, internallycirculating ventilation and externally-circulating ventilation. The working mechanisms given in Fig 2.

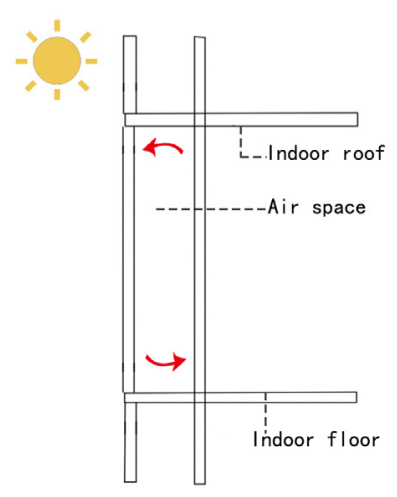

(a) Buffer-cavity DSF
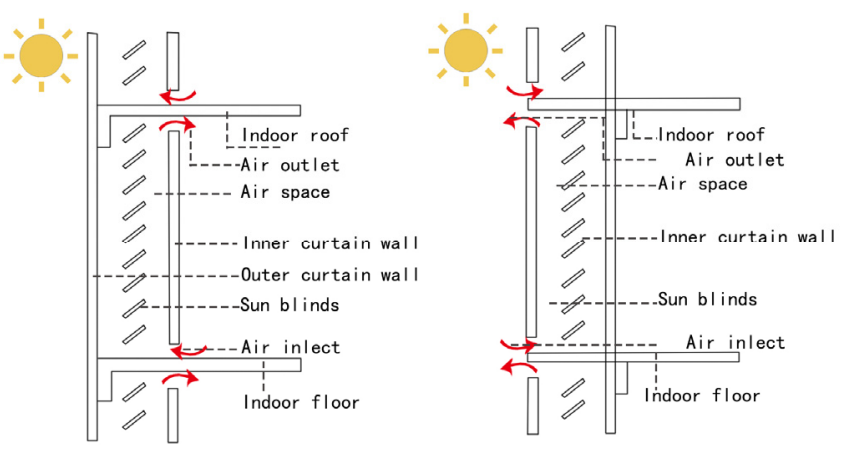

(b) Internally-circulating DSF

(c) Externally-circulating DSF

Fig. 2 Working mechanisms of DSFs

\subsection{Buffer-cavity DSFs}

The outer façade and inner facade of Buffer-cavity DFSs are composited with no devices of air inlet or air return, as well as the sunshade shutters; or the devices are closed in need. In winter, the air place is heated by solar radiation without external air flow, causing greenhouse effect, which improve the performance of heat preservation. However, in summer, the cavity may have the risk of overheating.

\subsection{Externally-circulating DSFs}

The inner curtain wall of externally-circulating DSFs is generally closed hollow glass, and the opening of the outer curtain wall is adjustable, and the single-layer glass is mostly used [4]. DSFs with external circulation rely on natural ventilation, so the maintenance and operation costs are low. It is a curtain wall form widely used. Here are the working mechanisms of the externally-circulating DSFs: (a). In summer: Put down the sun louvers to reduce the heat of solar radiation into the room; open the air inlet and outlet of the hot channel to form a through vertical channel, forming a "chimney effect", After the hot air rises, it is discharged through the top exhaust port to take away the heat in the hot aisle, which can effectively reduce the surface temperature of the inner curtain wall [5]. 


\subsection{Internally-circulating DSFs}

The outer curtain wall of internally-circulating DSFs are enclosed. The inner curtain wall adopts single-layer glass or single-layer aluminum doors and window; it needs to rely on mechanical system for ventilation, which has higher requirements for equipment. In summer, relying on mechanical means to draw indoor air into the hot aisle, using relatively low temperature indoor air to cool the air interlayer after absorbing solar radiation and lowering the air cavity Temperature[5].

\section{Researches on Thermal Performances}

Due to the structural characteristics of DSFs, such as the sandwich structure, the flexible device to manage air flow, the rotatable angle of the sunshade louvers et. Thus, DSFs have good thermal performance.

The thermal performance research of DSFs mainly focuses on energy consumption and operating principle, DSFs comprehensive heat transfer coefficient, DSFs internal chimney effect and operating principle, DSFs internal temperature field and flow field simulation calculation [5]. Rayment compared the performances of energy saving of different glazing, the result illustrated that double skin facades and heat reflecting glazing units obviously reduced energy consumption compared with single glazing . [6]. Grabe established a tool which chould quickly gain the design stratagems without use low efficiency CFD tools [7]. Zhu et al. established a physical model based on an internal breathing DSFs engineering example, simulated the comprehensive heat transfer coefficient of DSFs under different working conditions, and analyzed the wind speed, width and shading of the DSFs thermal channel The influence of louver position on the thermal performance of the envelope structure [8] Gratia et al. analyzed the influence of orientation, shading setting, shading equipment, inner skin color, solar radiation intensity, inner skin material, wind speed, curtain wall height and opening setting on its average temperature change, and the influence of orientation is more obvious [9]. Liu et al. used Fluent software to perform CFD numerical simulation, and compared the simulation results with actual test values. The simulation results are in good agreement with the actual test values [10].Bielek studied the method to heat recovery from the air exhaust for avoid too much heat reducing in winter season[11] .Through the application of CFD software, Shen Lingfeng calculated and analyzed the influence of the opening position and curtain wall spacing on the overall energy saving of Shanghai's first outer circulation DSFs.[12]. Yu Tianqi conducted a simulation study on the outer circulation DSFs of Beijing office buildings , founding that reducing the energy loss of the throughtype thermal bridge was the key to improving the energy-saving effect [3]. Preet et al.uesd forced ventilation to controll the heat ingress of PV-DSF, founding that the suitable combination of air place and air celocity could obviously reduce the solar radiation, and improving the efficiency of power ouput of the PV system[13] .

\section{Researches on Ventilation Performances}

Lu et al. used large eddy simulation method to simulate the ventilation performance of DSFs under different wind angles by CFD and compared them with wind tunnel test results [14]. Li et al. used ANSYS software to conduct a simulation study on the DSFs of the ward of Shanghai First People's Hospital, and found that natural ventilation is weak, and mechanical ventilation can be used to increase the ventilation effect and energy saving [15]. Ding et al. tested the internal ventilation and temperature of the DSFs of an existing reconstructed building in Chongqing, and pointed out the precautions in the design of the structural system [16]. Xu Xiaoli et al. simulated a louver by establishing a complex model in the CFD software The ventilation condition of the sunshade DS and the comparison of laboratory test results to confirm the accuracy of the model [17].Lei Miao found that the slat angle, air cavity thickness, and DSF height had different influences on the rate of the natural ventilated DSFs with venetian blinds [18]. Pourshab et al. found that the stack effect produced inside the cavity of double skin facades with horizontal and vertical louvers has enough power for air suction in the floors [19].Tao et al. analysed the airflow characteristics inside the NVDSF and found that the locations of window and dimensions of room make little effect on the ventilation rates, however ,the movements of air flow are significant to the air quality assessment[20].

\section{Discussions}

Although DSFs have many advantages in terms of energy saving and emission reduction, the construction and maintenance cost of DSFs is much higher than that of traditional glass curtain walls. The thermal performance of double skin facades has been established. According to the current situation in Shenzhen, the actual application of single-layer curtain walls and double skin facades have been analyzed, and the double skin facades are considered to be costly and economical [21].

However, in addition to the disadvantages of higher initial investment and cleaning costs, higher fire risk is also an inevitable risk. In the event of a fire, the hot channel between the inner and outer curtain walls can form a stack effect, and smoke and flames can easily spread to other parts of the building along the vertical channel. Since DSFs are a relatively new curtain wall technology after all, it is almost difficult to see clear regulations and requirements specifically for the fire protection design of DSFs in the fire code [2].

In case of fire, the fire protection design of DSFs should effectively prevent the smoke and flame from spreading upwards through the hot channel. In addition, 
the protection should ensure that the inner curtain wall of each layer above the fire protection layer would not break before the outer curtain wall, thus preventing the smoke and flame from damaging the inner curtain wall of non-fire floors and avoiding the fire spreading between upper and lower floors [2].

\section{Conclusions}

The unique structure of DSFs makes DSFs outstanding in terms of energy saving, ventilation, noise reduction, aesthetics, and comfort. However, DSFs also have some shortcomings: the initial investment and maintenance costs are relatively high, the fire protection design is relatively difficult, and the absence of industry standards. Therefore, DSFs are necessary to be estimated the overall energy-saving effect in advance. Research scholars should continue to in-depth optimization research on the engineering application value of DSFs, and ultimately bring good social and economic benefits.

\section{Acknowledgments}

This research is supported by grants from the Natural Science Foundation of Shandong Province (No. ZR201910280141).

\section{References}

1. Pomponi, F., et al.,Energy performance of DoubleSkin Façades in temperate climates: A systematic review and meta-analysis, Renewable and Sustainable Energy Reviews,54,2016)

2. Hang, Y.,Analysis of fire hazard of double-layer curtain wall based on geometric structure and ventilation mode,Fire Science and Technology,37(08),1079-1083(2018)

3. Tianqi, Y. and W. Yan,Study on energy consumption simulation of outer-circulation doubleglazed curtain wall,Building Energy Conservation,48(06),12-17+21(2020)

4. Lin, L., Research and analysis on the theory and engineering application of double-layer curtain walls at home and abroad,Green Building,10(04),3941(2018)

5. Cun, H., et al.,Research progress and prospects of double-layer thermal channel glass curtain wall,Environmental Engineering,33(S1),806811(2015)

6. Rayment, R.,Energy savings from sealed double and heat reflecting glazing units,Building Services Engineering Research \& Technology,10,1989)

7. Grabe, J.v.,A prediction tool for the temperature field of double facades Energy \& Buildings,34,2002)

8. Qingyu, Z., Du Guofu, and Z. Yu,CFD simulation calculation of comprehensive heat transfer coefficient of internally breathing glass curtain wall,HVAC,06,102-106(2005)
9. Gratia, E. and A.D. Herde,Greenhouse effect in double-skin facade Energy \& Buildings,39,2006)

10. Tao, L., G. Pingdao, and W. Shixiong,CFD numerical simulation of thermal performance of hot aisle glass curtain wall, Journal of Donghua University (Natural Science Edition),04,486489(2008)

11. Bielek, B., J. Klem, and M. Macák,Physical Cavity of a Double Skin Facade as a Source of Pre-Heated Air in the Winter Season for the Heat Recovery Unit of a Facade,Slovak Journal of Civil Engineering,27(4),7-10(2019)

12. Lingfeng, S. and L. Peng, Research on energy saving of outer circulation double-layer curtain wall,Clean and Air Conditioning Technology,01,45-48(2015)

13. Preet, S., M.K. Sharma, and J. Mathur,Performance evaluation of Photovoltaic double-skin facade with forced ventilation in the composite climate,Journal of Building Engineering,32(prepublish),2020)

14. Dan, L., et al., Numerical simulation study on ventilation performance of double-curtain wall buildings,Journal of Zhejiang University (Engineering Science Edition),01,47-51(2005)

15. Li Rongmin and G. Jianming,Simulation and analysis of airflow organization in the hot aisle of glass curtain wall, Heating Ventilation and Air Conditioning,01,23-28(2007)

16. Yong, D., L. Baizhan, and L. Hong,Measurement and analysis of the ventilation effect of a doubleskinned envelope structure in Chongqing,Heating Ventilation and Air Conditioning,08,42-45(2007)

17. $\mathrm{Xu}, \mathrm{X} .-1$. and $\mathrm{Z}$. Yang,Natural ventilation in the double skin facade with venetian blind,Energy \& Buildings,40,2008)

18. Miao, L. and C.-L. Chow, Investigation of burning photovoltaic panels on a double-skin facade with ejecting flame from an adjacent room fire Indoor and Built Environment,28,2019)

19. Pourshab, N., M.D. Tehrani, and D. Toghraie,Application of double glazed façades with horizontal and vertical louvers to increase natural air flow in office buildings,Energy \& Buildings,C,200(2020)

20. Yao, T., et al.,Ventilation performance of a naturally ventilated double-skin façade in buildings, Renewable Energy,200(C),2020)

21. Lieyu, H., The influence of single-layer or DSFs in high-rise office buildings on air-conditioning load,Architecture Science,14(4),29-34(1998) 